Check for updates

Cite this: Phys. Chem. Chem. Phys., 2017, 19, 24605

Received 1st March 2017, Accepted 12th May 2017 DOI: 10.1039/c7cp01341h

rsc.li/pccp

\section{Imaging on-surface hierarchical assembly of chiral supramolecular networks $\dagger$}

\author{
Laerte L. Patera, (D) *abc Zhiyu Zou, ${ }^{\text {ad }}$ Carlo Dri, (D) ${ }^{\text {ace }}$ Cristina Africh, (D) ${ }^{\text {a }}$ \\ Jascha Repp ${ }^{\mathrm{b}}$ and Giovanni Comelli (D) ${ }^{\text {ac }}$
}

\begin{abstract}
The bottom-up assembly of chiral structures usually relies on a cascade of molecular recognition interactions. A thorough description of these complex stereochemical mechanisms requires the capability of imaging multilevel coordination in real-time. Here we report the first direct observation of hierarchical expression of supramolecular chirality at work, for 10,10'-dibromo-9,9'-bianthryl (DBBA) on $\mathrm{Cu}(111)$. Molecular recognition first steers the growth of chiral organometallic chains and then leads to the formation of enantiopure islands. The structure of the networks was determined by noncontact atomic force microscopy (nc-AFM), while high-speed scanning tunnelling microscopy (STM) revealed details of the assembly mechanisms at the ms time-scale. The direct observation of the chirality transfer pathways allowed us to evaluate the enantioselectivity of the interchain coupling.
\end{abstract}

\section{Introduction}

Chirality recognition is essential in many biological systems, determining optical and pharmaceutical properties of enantiopure compounds. ${ }^{1,2}$ Furthermore, the realization of chiral catalysts, which allows the production of non-racemic chemicals with high enantioselectivity, has become of paramount importance in modern synthetic chemistry. ${ }^{3,4}$ However, the mechanisms underlying the selectivity of such processes remain poorly understood ${ }^{5,6}$ limiting industrial applications. ${ }^{7,8}$

In the field of supramolecular chemistry, it was shown that chirality can be expressed from single molecules to mesoscopic systems, by means of non-covalent assemblies. ${ }^{9-12}$ Direct observations, provided by in situ STM, highlighted the importance of dynamic mutual conformational adjustments for the chiral recognition to occur. ${ }^{13}$ In similar ways, exploiting recognition processes, enantiopure phases can be successfully synthesized on metal surfaces. ${ }^{14-16}$ Moreover, since the control of the supramolecular assembly critically depends on the interplay between molecule-molecule and molecule-substrate interactions, ${ }^{17-21}$ different strategies were developed for the realization of extended chiral networks, exploiting different molecular structures ${ }^{22,23}$ and bonding motifs. ${ }^{13,24-27}$

\footnotetext{
${ }^{a}$ IOM-CNR Laboratorio TASC, Area Science Park, 34149 Trieste, Italy

${ }^{b}$ Institute of Experimental and Applied Physics, University of Regensburg,

93053 Regensburg, Germany. E-mail: laerte.patera@ur.de

${ }^{c}$ Department of Physics, University of Trieste, 34127 Trieste, Italy

${ }^{d}$ Abdus Salam International Centre for Theoretical Physics, 34151 Trieste, Italy

${ }^{e}$ Elettra - Sincrotrone Trieste S.C.p.A., 34149 Trieste, Italy

$\dagger$ Electronic supplementary information (ESI) available. See DOI: 10.1039/c7cp01341h
}

Recently, special attention has been devoted to systems presenting a hierarchical chirality transfer, which reveals the presence of sequentially-activated enantioselective mechanisms. ${ }^{24,28-35}$ Blüm et al. ${ }^{28}$ reported the fascinating case of rubrene $\left(\mathrm{C}_{42} \mathrm{H}_{28}\right)$ assembling into pentagonal structures, which act as building blocks for a second generation decagons. Interestingly, both the resulting supramolecular structures are enantiopure, indicating the conservation of chirality during the coordination. Hierarchical organization can also be steered through metal-ligand interactions, where the presence of metal adatoms imposes distinctive multilevel binding processes. ${ }^{24,33}$ Furthermore, sequential self-assembly of nanoporous homochiral networks has been recently observed using porphyrin derivatives, ${ }^{29}$ representing an appealing platform for the realization of flexible functional materials. However, the atomic-scale mechanisms driving molecular recognition in hierarchical assemblies are yet to be clarified and the factors governing the enantioselectivity of these processes are largely unknown, limiting the possibility to design a priori tailored molecular units with programmed functionalities.

In this work, we report the study of chirality expression during 10,10'-dibromo-9,9'-bianthryl (DBBA) self-assembly on $\mathrm{Cu}(111)$, combining state of the art scanning probe microscopy (SPM) techniques. In fact, despite DBBA has been extensively studied on the $\mathrm{Cu}(111)$ surface as precursor for the on-surface synthesis of chiral graphene nanoribbons, ${ }^{36-38}$ little attention has been devoted to the molecular structure of Ullmann intermediates. In particular, the chiral properties of such compounds have not been properly addressed so far.

Upon deposition at $300 \mathrm{~K}$, the molecules undergo debromination and subsequent assembly into chiral organometallic chains, which further interact forming 2D enantiopure islands. 
The atomic configurations of the resulting compounds are determined by means of high-resolution noncontact atomic force microscopy (nc-AFM), while high-speed scanning tunnelling microscopy (STM) measurements shed light on the assembly mechanisms, revealing the presence of co-existing coordination pathways. Finally, exploiting time-resolved STM sequences, the enantioselectivity of the interchain coupling has been evaluated.

\section{Experimental}

Experiments were carried out in two UHV systems, equipped with standard sample preparation facilities. $\mathrm{Cu}(111)$ single-crystal samples were cleaned by several sputtering $\left(\mathrm{Ar}^{+} / \mathrm{Ne}^{+}, 1 \mathrm{keV}\right)$ and annealing (773 K) cycles.

Variable-temperature experiments were carried out with an Omicron VT-STM. High-speed STM measurements were performed using FAST, ${ }^{39}$ an add-on module for increasing the imaging frequency of commercial scanning probe microscopes up to video-rate. ${ }^{40}$ In the experiments here discussed, image time-series were acquired in quasi-constant height mode with frame rates between 10 and $36.5 \mathrm{~Hz}$. The high-speed images are presented with horizontal fast scanning direction. Bias voltages are given as sample bias with respect to the tip. 10,10'-dibromo9,9'-bianthryl molecules (AOKBIO, 98+\% purity) were sublimed onto the sample held at $\cong 300 \mathrm{~K}$.

Low-temperature experiments were carried out with a homebuilt combined scanning tunnelling/atomic force microscope, at a temperature of $6.8 \mathrm{~K}$. The atomic force microscope's qPlus tuning fork ${ }^{41}$ was operated in the frequency modulation mode with sub-angstrom oscillation amplitudes. ${ }^{42}$ Bias voltages are given as sample bias with respect to the tip. A small amount of CO was dosed onto the surface for tip functionalization. ${ }^{43} \mathrm{NaCl}$ islands were grown to facilitate the pick-up of $\mathrm{CO}$ molecules to the tip apex from these islands. AFM data was obtained with a CO-terminated tip apex. 10,10'-dibromo-9,9'-bianthryl molecules (AOKBIO, 98+\% purity) were sublimed onto the sample kept at $\cong 8 \mathrm{~K}$ inside the microscope and subsequently annealed at $\cong 300 \mathrm{~K}$ in the preparation chamber.

\section{Results and discussion}

In gas-phase, DBBA is not planar, due to steric hindrance, presenting a large angle between the anthracene moieties (see Fig. 1), which confers axial chirality to the molecule. Upon surface deposition, the molecules remain non-planar, resulting in racemic ad-layers of two enantiomers, named $\alpha$ and $\beta$ in Fig. 1. Moreover, on $\mathrm{Cu}(111)$, at surface temperatures above $240 \mathrm{~K}$, debromination spontaneously occurs, ${ }^{36}$ generating biradical active species. While on $\mathrm{Au}(111)$ covalent coupling is readily triggered after the radical generation, ${ }^{44,45}$ for the case of similar molecules on $\mathrm{Cu}(111)$, metal adatoms have been shown to bind to the active $\mathrm{C}$ atoms, giving rise to $\mathrm{C}-\mathrm{Cu}-\mathrm{C}$ coordination (see Fig. 1), ${ }^{46-48}$ recently identified as possible intermediates of the Ullmann coupling reaction. ${ }^{46,49}$

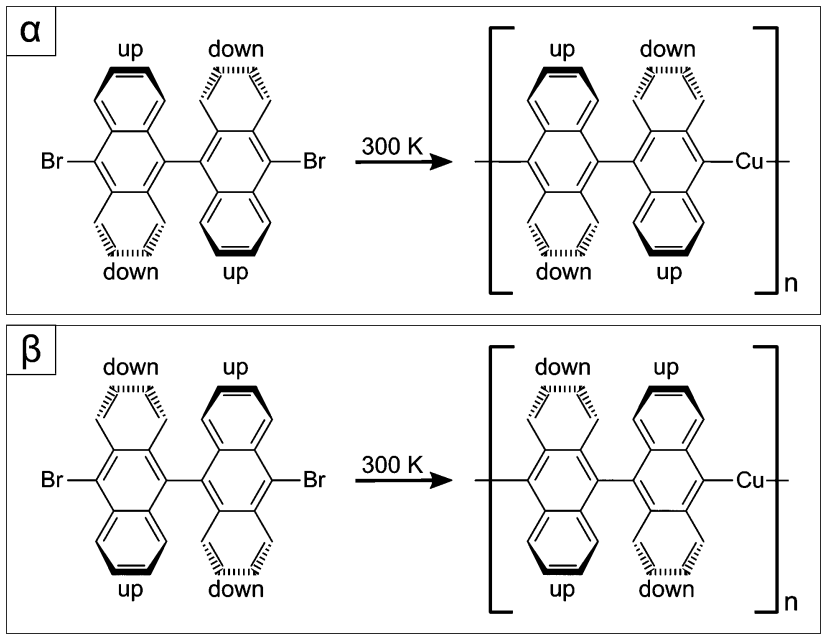

Fig. 1 Reaction scheme for the formation of chiral organometallic chains from DBBA enantiomers on $\mathrm{Cu}(111)$. Top (bottom) panel corresponds to $\alpha(\beta)$ enantiomer

Fig. 2a shows a STM image of the $\mathrm{Cu}(111)$ surface after DBBA deposition at $300 \mathrm{~K}$. Molecular self-assembly gives rise to ordered zigzag-like chain structures, which can further coalesce into 2D islands. The absence of moiré patterns in uniform structures, exceeding $10 \mathrm{~nm}$ in length, suggests that the molecular orientation with respect to the substrate provides commensurability to the chains.

Around room-temperature (RT), the molecular assembly presents equilibrium fluctuations, due to the balance between energy minimization from chain coordination and loss of conformational and translational entropy, ${ }^{50,51}$ resulting in the continuous diffusion of single molecules and chains (indicated by the fuzzy regions in Fig. 2a). As previously reported by Wintterlin et al., ${ }^{50}$ this fact, along with the absence of qualitative structural evolution over long periods, reveals that the system has reached thermodynamic equilibrium.

In order to clarify the molecular organization within the chains, such structures have been characterized by means of nc-AFM with a CO-functionalized tip. ${ }^{43,52}$ Since the molecules are not planar, it is not possible to fully resolve their structure by constant-height AFM imaging. ${ }^{53}$ However, the image in Fig. $2 \mathrm{~b}$ reveals intramolecular features of a dimer (indicated by arrows on a molecule in Fig. 2b), which allow resolving the geometric arrangement of the chemical bonds located at the two protruding lobes of the molecule. In Fig. $2 \mathrm{~b}$, due to the non-planar geometry of the bi-anthracene, the protruding lobes (indicated by arrows in Fig. $2 \mathrm{~b}$ and c) exhibit repulsive (less negative $\Delta f$ ) contrast in the AFM images, while the parts closer to the substrate are not visible. By superimposing a model on the image and rotating in-plane the anthracene units until the model fits the bright features in the AFM image, the orientation of the molecule can be determined (see Fig. S1 in the ESI $\dagger$ ). In this way, we extracted an angle of about $82^{\circ}$ between the azimuthal orientation of the anthracene long axis and the chain direction (see Fig. S1 and S2c in the ESI $\dagger$ ). This observation indicates that the molecular coordination involves aryl group interaction, in agreement with 

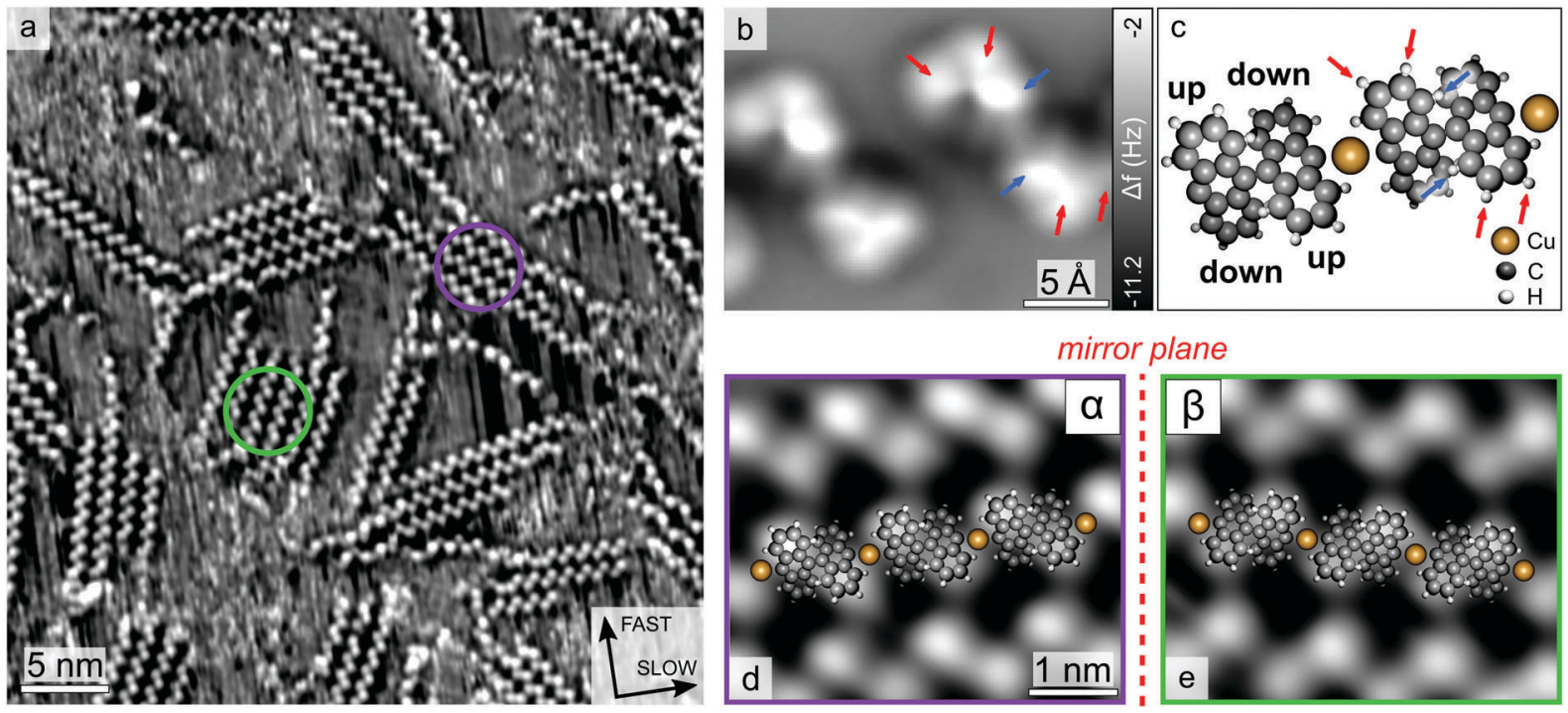

Fig. 2 DBBA self-assembly on Cu(111). (a) STM image of molecular chains and islands [-1 V, $0.5 \mathrm{nA}, 295 \mathrm{~K}, 60 \mathrm{~s}$ per frame]. Circles highlight domains with opposite chirality. Fast and slow scanning directions are specified. (b) nc-AFM image of a dimer obtained with a CO tip [ $\Delta z=2.9 \AA$, $6.8 \mathrm{~K}, \mathrm{STM}$ setpoint: $1 \mathrm{~V}, 1.4 \mathrm{pA}$. Arrows indicate the main intra-molecular features. (c) 3D model of the molecular structure imaged in (b). The arrows specified in (b) are superimposed. ( $d$ and e) Zoom-in on two regions of different chirality, extracted from the STM image in (a), overlaid with the proposed structures. A dashed line between the panels indicates the mirror symmetry between $\alpha$ and $\beta$ domains (images were rotated to display the mirror plane along the vertical direction).

previously proposed models based on STM images. ${ }^{36}$ However, the distance between the molecules $(1.2 \mathrm{~nm})$ is larger than the one found in anthracene chains on $\mathrm{Au}(0.85 \mathrm{~nm}),{ }^{44}$ suggesting the presence of bridging $\mathrm{Cu}$ adatoms, ${ }^{36,38}$ usually not visible in STM images. ${ }^{54-56}$ Since the adatoms are expected to sit closer to the substrate than the molecular protruding lobes, they cannot be detected by constant-height AFM. Moreover, the observed distorted shape of diffusing chains (see below) suggests an important intermolecular interaction, supporting metal-organic coordination. Fig. 2c shows the resulting structural model of the organometallic chain, in line with recent results presented by Schulz et al..$^{38}$ In Fig. 2b, arrows indicate the protruding $\mathrm{C}-\mathrm{H}$ bonds within the molecule. The bright features located closer to the centre of the molecule (see blue arrows in Fig. $2 \mathrm{~b}$ and c) correspond to bonds pointing out-of-plane due to steric hindrance, more than the other visible $\mathrm{C}-\mathrm{H}$ bonds (indicated by red arrows in Fig. $2 \mathrm{~b}$ and c). Instead, the ones adjacent to the aryl groups cannot be distinguished, indicating a different height with respect to the substrate, as result of a lateral bending of the anthracene towards the $\mathrm{Cu}$ adatoms. A slight difference between the protruding lobes of the single molecule can be observed in the AFM image (see Fig. 2b), revealing an asymmetry in the adsorption configuration, reflected also in some STM images (see below). This effect may be attributed to the possible presence of $\mathrm{Br}$ atoms underneath the tilted anthracene units, as reported by Bronner et $a .^{57}$

Concerning the expression of supra-molecular chirality, a closer inspection reveals that the chains exhibit a high enantiopurity, indicating the formation of 1D chiral organometallic structures. Interestingly, also the islands are usually composed exclusively of either $\alpha$ or $\beta$ enantiomers, as shown in Fig. 2 . In fact, upon deposition at $300 \mathrm{~K}$, the concentration of chiral defects is less than $10 \%$, revealing that chirality is preserved in the $2 \mathrm{D}$ molecular assembly. In fact, the chains in the molecular islands are found to be aligned only along two groups of threefold symmetric directions (see Fig. S3 in the ESI $\dagger$ ), ${ }^{37}$ which indicates the presence of two distinguished inequivalent phases (i.e. $\alpha$ and $\beta$ ). This differs from the self-assembly behaviour of methylthiolate complexes on $\mathrm{Au}(111),{ }^{58}$ where, in order to reduce the steric repulsion, chains of alternating chirality couple, leading to the formation of racemic islands. In contrast, in our case, molecular recognition mechanisms steer a hierarchical transfer of chirality from molecules to chains and from chains to islands, suggesting the presence of enantioselective assembly processes acting at different scales.

To study the self-assembly mechanisms, we investigated the time-evolution of the molecular network at temperatures between 290 and $370 \mathrm{~K}$. Under these conditions, the assembly processes are too fast to be properly imaged through conventional STM. For this reason, we exploited the high-speed imaging capability provided by the FAST module, which allows acquiring STM time-series up to video-rate. ${ }^{39}$ In this way, it is possible to resolve in real-time chemical reactions steps with atomic resolution. ${ }^{59,60}$

Fig. 3 shows the three assembly mechanisms captured by high-speed STM imaging. The process in Fig. 3a and b advances by attachment of a single enantiomer to an organometallic chain. The centre of Fig. 3a presents a chain composed of four molecules. After $108 \mathrm{~ms}$ (Fig. 3b), an additional enantiomer of the same chirality is found to be attached at the termination on the bottom. This process involves the formation of organometallic bonds and leads to the growth of enantiopure 1D structures. A second mechanism is also characterized by organometallic coordination, where an individual molecule attaches to a chain 

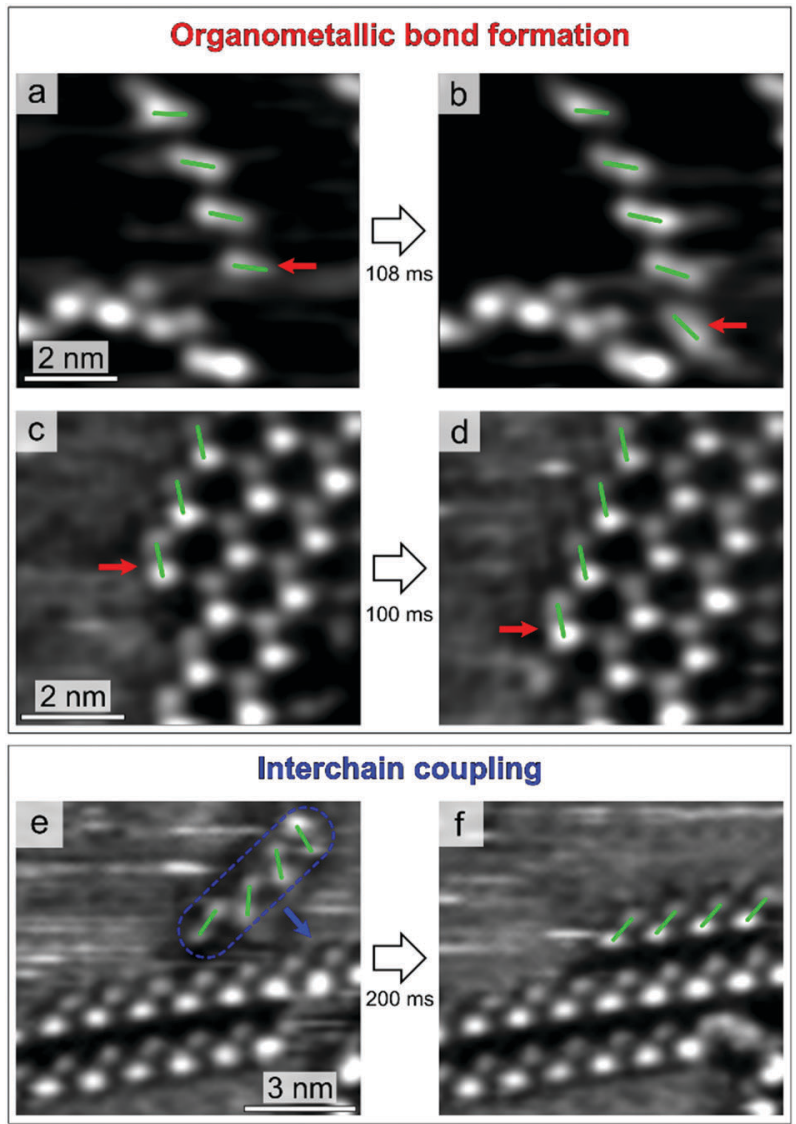

Fig. 3 Self-assembly mechanisms. ( $a$ and b) Enantiomer attachment to chain termination [-1 V, $1.4 \mathrm{nA}, 294 \mathrm{~K}, 27 \mathrm{~ms}$ per frame]; (c and d) enantiomer attachment to the kink of island edge [ $-1.5 \mathrm{~V}, 0.3 \mathrm{nA}, 343 \mathrm{~K}, 100 \mathrm{~ms}$ per frame]. In each frame, relevant molecules are highlighted with coloured dashes and red arrows highlight the position of the enantiomer located at the end of the chain. (e and f) Interchain coupling [-2 V, 0.5 nA, $295 \mathrm{~K}, 100 \mathrm{~ms}$ per frame]. Blue arrow indicates the chain attachment. The difference in the monomer appearance between $a$ and $b$ and $c-f$ panels is likely due to the tip-dependent STM contrast.

located at the edge of a 2D island (Fig. 3c and d). Fig. 3(e and f) shows yet another process, involving coupling between preformed organometallic chains. In the STM sequence, the chain first approaches and then attaches to the island edge. Due to the lack of lateral functional groups, such interchain coupling is expected to be mediated by van der Waals forces. ${ }^{61}$
All the mechanisms are highly enantioselective and work identically for both molecular chiralities. Concerning the organometallic coordination, the clear correlation between molecular chirality and chain orientation with respect to the $\mathrm{Cu}(111)$ crystallographic directions (see Fig. S3 in the ESI $\dagger$ ) indicates an important molecule-substrate interaction, ${ }^{36,37}$ to which we attribute the main reason for the high enantioselectivity of the organometallic coordination process. In fact, the rather strong coupling with the substrate does not allow heterochiral molecules to attach to the chains, while still maintaining a favourable substrate adsorption site. These arguments also explain why defect formation has been observed only for the radical attachment to single chain (see Fig. S2 in the ESI $\dagger$ ), but not through its coordination at island edges. In fact, as compared to the extended islands, single chains can access a larger number of adsorption configurations, owing to their higher surface mobility, allowing for the attachment of radicals with opposite chirality.

Conversely, the formation of enantiopure islands is given by two distinct contributions: attractive van der Waals interactions between the chains and molecule-substrate adsorption energy.

While the former leads to the formation of $2 \mathrm{D}$ networks, steering the interchain coupling, the latter stabilizes the 1D organometallic structures along specific directions according to their chiralities, ${ }^{37}$ thereby providing enantioselectivity to the process.

Concerning the nature of the assembly processes, qualitative considerations can be done based on the observed event rates. Above RT, all the mechanisms shown in Fig. 3 are found to be reversible, i.e. it is possible to observe both formation and breaking of the molecular connections. However, the one shown in Fig. 3e and $\mathrm{f}$ exhibits a considerably higher disruption rate, compared to the first two processes, indicating that interchain coupling is less stable than organometallic bonding. This difference in the coordination stability is responsible for the general hierarchical behaviour of the self-assembly.

In order to shed light on the hierarchical chirality transfer, we investigated in detail the interchain coupling process, since it guides the coordination of $1 \mathrm{D}$ chiral structures, giving rise to second-generation enantiopure networks.

Fig. 4a shows three chiral domains, one composed of $\alpha$ enantiomers and two of $\beta$ ones. In Fig. $4 \mathrm{~b}$, a $\beta$ chain (four
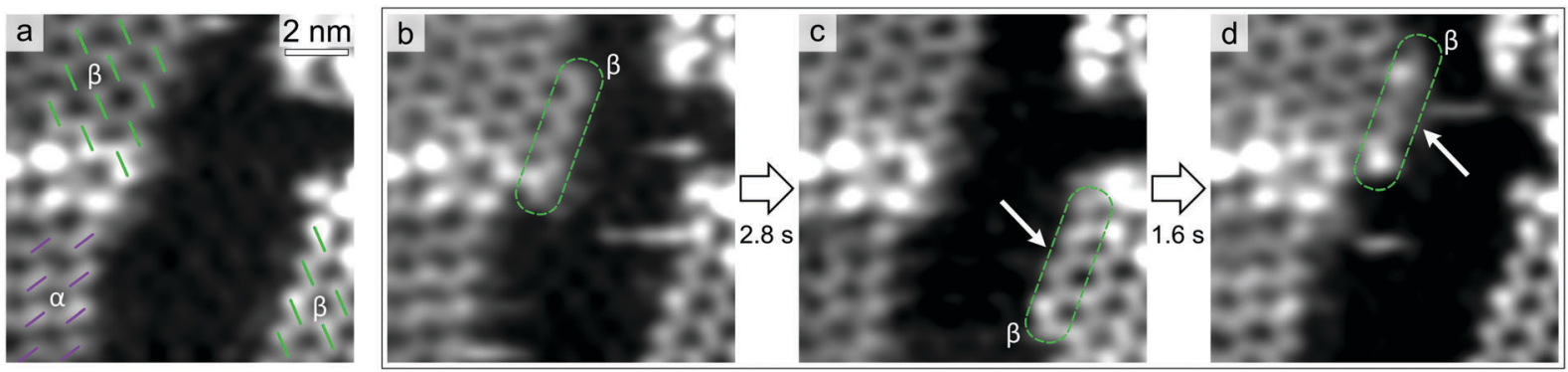

Fig. 4 Enantioselectivity of interchain coupling. (a) STM image of three homochiral domains. The molecules are highlighted with coloured dashes according to their chirality. (b-d) Selected STM frames from a time-series. The exchanged chain is indicated in green. Arrows serve as a guide to the eye. [-1.6 V, $0.22 \mathrm{nA}, 297 \mathrm{~K}, 100 \mathrm{~ms}$ per frame]. 
molecules long, highlighted in green) has attached to the $\beta$ domain edge in the top-left corner. In Fig. 4c and d, this chain first moves to the $\beta$ edge in the bottom-right (Fig. 4c), then goes back to the initial one (Fig. 4d). Interestingly, within the timescale of STM measurements (100 ms per frame), exchange events of the $\beta$ chain appear to occur between $\beta$ island edges only. In an extended STM time-series $(\approx 15 \mathrm{~s})$, we observed the chain attaching/detaching for 16 times at the $\beta$ molecular island, while no detectable heterochiral coupling event can be clearly identified. This indicates a remarkable preference for homochiral coupling to occur, favouring the formation of enantiopure islands.

To better understand the molecular recognition interaction, we measured the residence times of different chains along the chiral edges. Fig. 5(a and c) shows two stable molecular islands (left: $\beta$ island, right: $\alpha$ island), with two short oligomers of the opposite chirality moving in the separating region: a chain with three $\alpha$ enantiomers (marked with violet dashes in Fig. 5a) and one composed of three $\beta$ ones (marked with green dashes in Fig. 5c). At RT, such short chains exhibit remarkable dynamics, resulting in a fast attachment/detachment process (see Movies in ESI†). For both the cases shown in Fig. 5(a and c), the chain position along the different molecular edges has been tracked

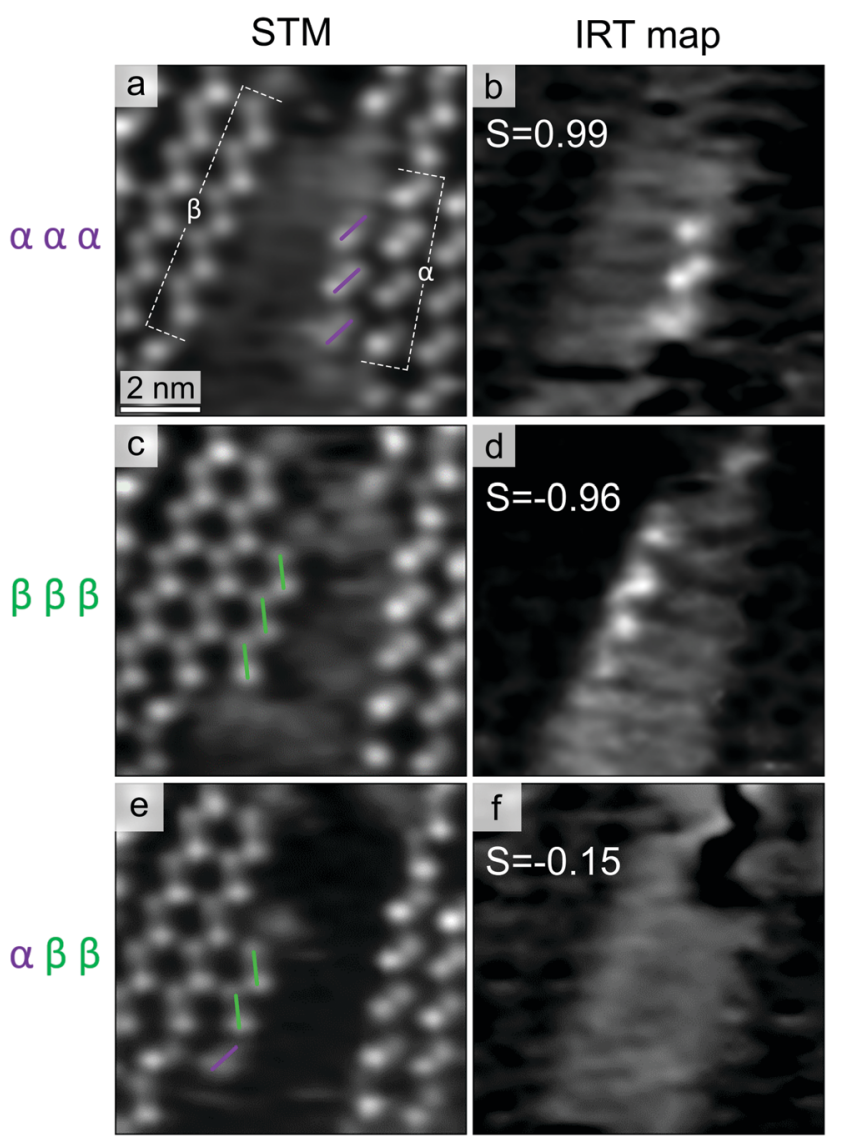

Fig. 5 Enantioselectivity of interchain coupling. (a, c and e) STM frames extracted from extended movies acquired on the same region, for the case of: $\alpha \alpha \alpha, \beta \beta \beta$ and $\alpha \beta \beta$ chains, respectively. (b, $d$ and f) IRT maps obtained from 36 consecutive frames ( $\approx 1 \mathrm{~s}$ ) [-1 V, $1.4 \mathrm{nA}, 294 \mathrm{~K}, 27 \mathrm{~ms}$ per frame]. by acquiring STM time-series with high temporal resolution (27 ms per frame). Despite the high mobility of the short chains, which prevents us from discriminating each exchange event, it is possible to quantify the probability to find the chain along the two edges, through the measurement of integrated residence time (IRT). Fig. 5(b and d) shows the average on 36 consecutive frames, after the subtraction of a background image (where the diffusing chain was not present), in which the preferential position of the moving chains turns to be enhanced compared with individual frames. In these images, the grey-scale contrast represents the IRT of the chain configurations with molecular resolution. Comparing the IRT intensities along the different island edges, it is possible to quantify the enantioselectivity of the interchain coupling process. For this purpose, we introduce the parameter $S$, which expresses the difference between IRT along the opposite homochiral edges:

$$
S=\frac{I_{\alpha}-I_{\beta}}{I_{\alpha}+I_{\beta}}
$$

where $I_{\alpha}$ and $I_{\beta}$ are the integrated intensities along the island edges with respect to a background level (see Fig. S4 in the ESI $\dagger$ for details). In presence of a non-selective process, $S$ drops to zero, while it reaches values close to \pm 1 for highly selective mechanisms. Positive (negative) values of $S$ express a preferential chain distribution along the $\alpha(\beta)$ edge. Since the STM sequences shown in Fig. 5 have been acquired on the same surface region and for chains composed of the same number of molecules, it is possible to compare the interchain coupling selectivity.

For both the enantiopure chains, the main IRT features are localized along the edge of the same chirality, revealing a negligible adsorption along the heterochiral edge. $S$ values of 0.99 and -0.96 indicate the high enantioselectivity of the interchain homocoupling process. Since the two configurations are symmetric due to geometrical reasons, the difference between the two reported absolute values of $S$ is within the experimental error.

It is worth noting that, at the thermodynamic equilibrium, the residence-time distribution of different configurations reflects the relative binding energy difference. As mentioned above, for the system considered in this study, above RT, the large structural fluctuations combined with a lack of qualitative evolution over long times (up to few days) indicate that the equilibrium condition has been reached. ${ }^{50,51,62}$ This allows exploiting the IRT maps to estimate the binding energy difference between the homo- and heterochiral configurations. In fact, assuming the activation entropy to be the same for the two attachment processes, the ratio of the probability distribution can be expressed as:

$$
\frac{p_{\alpha}}{p_{\beta}}=\frac{I_{\alpha}}{I_{\beta}}=\exp \left(-\frac{\Delta E_{\mathrm{b}}}{k_{\mathrm{B}} T}\right),
$$

where $p_{\alpha}$ and $p_{\beta}$ are the probabilities of coupling of a specific ( $\alpha$ or $\beta$ ) chain along the $\alpha$ and $\beta$ edges, $\Delta E_{\mathrm{b}}$ is the binding energy difference between the two chain configurations, $k_{\mathrm{B}}$ is the Boltzmann constant, $T$ is the surface temperature and the same pre-exponential in the Arrhenius equation for both the 
processes is assumed. By using this expression, for the homochiral $\alpha$ and $\beta$ chains composed of three molecules shown in Fig. 5(a and c), we obtained $\Delta E_{\mathrm{b}}$ values of 153 and $95 \mathrm{meV}$, respectively. Since, as observed above, the two configurations are practically equivalent, these values reflect the uncertainty of the real binding energy difference, which we therefore estimate to be around $120 \mathrm{meV}$. This value supports the assumption that interchain coupling is governed by van der Waals forces, as typical values of pair-wise interaction in noble gases lie between 15 and $35 \mathrm{meV},{ }^{63}$ in line with the estimate we obtain for a threemolecule chain. While, due to the experimental error quoted above, this estimate is only indicative and should be considered with care, it is interesting to note that from measurements of this kind, values of the relevant thermodynamic parameters can be derived.

To further corroborate this argument, we examine the case of non-enantiopure oligomers. In Fig. 5e, the chain is composed of one $\alpha$ and two $\beta$ molecules (details about the formation of defective organometallic chains are provided in the ESI $\dagger$ ). In the corresponding IRT map (Fig. 5f), the absence of well-defined features along both island edges $(S=-0.15)$ reveals a poor chain adsorption stability, highlighting how, for the case of short chains, the enantioselectivity of the process is importantly affected by the presence of chiral defects. This is in line with the thermodynamic control of the process selectivity. In fact, the (small) negative $S$ value implies a higher adsorption binding energy along the $\beta$ edge (in the order of $10 \mathrm{meV}$ ), which is expected given relative $\beta$ excess in the chain composition (two $\beta$ $v s$. one $\alpha$ ). Despite the fact that the experimental uncertainty does not allow for a quantitative comparison between the homoand heterocoupling energy gains, the negative $S$ value indicates that, also for the case of non-enantiopure chains, the interaction is driven by thermodynamic arguments, steering the homochiral coupling.

Concerning the kinetic factors involved in the interchain coupling mechanism, in principle, relevant information can be obtained by measuring the attachment/detachment rates, since they are related to the difference in potential energy barriers between the homo- and heterocoupling configurations. However, the residence times along heterochiral edges are usually below our temporal resolution, preventing us from discriminating individual exchange events. Nevertheless, in the IRT maps of Fig. 5(b and d), the lack of a depletion region close to the heterochiral edge in the IRT maps suggests that the approaching barrier, if present, is at most comparable to the diffusion ones.

\section{Conclusions}

In summary, combining high-resolution nc-AFM and variabletemperature high-speed STM, we revealed the structures and self-assembly mechanisms of chiral organometallic networks on $\mathrm{Cu}(111)$. The molecule-substrate interaction is found to contribute importantly to the assembly enantioselectivity, dictating specific chirality-dependent orientations of the molecules. Through real-space imaging of molecular coordination at the ms time-scale, we observed hierarchical chirality transfer processes for the first time. By monitoring the molecular kinetics, we were able to evaluate the enantioselectivity of interchain coupling, providing information about energy gains and kinetic barriers involved in the chirality recognition process. These results represent a significant step forward in the understanding of enantioselective self-assembly, demonstrating the feasibility of monitoring complex chirality transfer mechanisms in real-time.

\section{Acknowledgements}

We thank G. Troiano, S. Schillani, D. Giuressi, G. Cautero, P. Scheuerer, F. Albrecht and F. Berti for fruitful discussion and help. This project has received funding from the EU-H2020 Research and Innovation programme under grant agreement no. 654360 NFFA-Europe. Financial support from the Marie Curie Initial Training Network "MOLESCO" (no. 606728) is gratefully acknowledged. We thank Dr Friedrich Esch for his collaboration in the development and optimization of the Fast scanning module. Z. Z. acknowledges support by the "ICTP TRIL Programme, Trieste, Italy" in the framework of the agreements with the Elettra and CNR-IOM laboratories.

\section{References}

1 T. Verbiest, S. Van Elshocht, M. Kauranen, L. Hellemans, J. Snauwaert, C. Nuckolls, T. J. Katz and A. Persoons, Science, 1998, 282, 913-915.

2 J. McConathy and M. J. Owens, Prim. Care Companion J. Clin. Psychiatry, 2003, 5, 70-73.

3 T. Mallat, E. Orglmeister and A. Baiker, Chem. Rev., 2007, 107, 4863-4890.

4 M. Heitbaum, F. Glorius and I. Escher, Angew. Chem., Int. Ed., 2006, 45, 4732-4762.

5 J. R. Cronin and S. Pizzarello, Science, 1997, 275, 951-955.

6 D. G. Blackmond, Cold Spring Harbor Perspect. Biol., 2010, 2, 1-17.

7 Z. Wang, K. Ding and Y. Uozumi, Handb. Asymmetric Heterog. Catal., 2008, 1-24.

8 H. U. Blaser, F. Spindler and M. Studer, Appl. Catal., A, 2001, 221, 119-143.

9 J. Weckesser, A. De Vita, J. V. Barth, C. Cai and K. Kern, Phys. Rev. Lett., 2001, 87, 96101.

10 S. Blankenburg and W. G. Schmidt, Phys. Rev. Lett., 2007, 99, 196107.

11 U. Schlickum, R. Decker, F. Klappenberger, G. Zoppellaro, S. Klyatskaya, W. Auwärter, S. Neppl, K. Kern, H. Brune, M. Ruben and J. V. Barth, J. Am. Chem. Soc., 2008, 130, 11778-11782.

12 R. Fasel, M. Parschau and K.-H. Ernst, Nature, 2006, 439, 449-452.

13 M. Lingenfelder, G. Tomba, G. Costantini, L. C. Ciacchi, A. De Vita and K. Kern, Angew. Chem., Int. Ed., 2007, 46, 4492-4495. 
14 A. Schiffrin, A. Riemann, W. Auwärter, Y. Pennec, A. WeberBargioni, D. Cvetko, A. Cossaro, A. Morgante and J. V. Barth, Proc. Natl. Acad. Sci. U. S. A., 2007, 104, 5279-5284.

15 A. Kühnle, T. R. Linderoth, B. Hammer and F. Besenbacher, Nature, 2002, 415, 891-893.

16 R. Fasel, M. Parschau and K.-H. Ernst, Angew. Chem., Int. Ed., 2003, 42, 5178-5181.

17 J. V. Barth, J. Weckesser, G. Trimarchi, M. Vladimirova, A. De Vita, C. Cai, H. Brune, P. Günter and K. Kern, J. Am. Chem. Soc., 2002, 124, 7991-8000.

18 S. De Feyter, A. Gesquiere, F. De Schryver, C. Meiners, M. Sieffert and K. Mullen, Langmuir, 2000, 16, 9887-9894.

19 R. Otero, M. Schöck, L. M. Molina, E. Lægsgaard, I. Stensgaard, B. Hammer and F. Besenbacher, Angew. Chem., Int. Ed., 2005, 44, 2270-2275.

20 A. Kühnle, T. R. Linderoth and F. Besenbacher, J. Am. Chem. Soc., 2006, 128, 1076-1077.

21 A. Kühnle, T. R. Linderoth and F. Besenbacher, J. Am. Chem. Soc., 2003, 125, 14680-14681.

22 C. Bombis, S. Weigelt, M. M. Knudsen, M. Nørgaard, C. Busse, E. Lægsgaard, F. Besenbacher, K. V. Gothelf and T. R. Linderoth, ACS Nano, 2010, 4, 297-311.

23 S. Stepanow, N. Lin, F. Vidal, A. Landa, M. Ruben, J. V. Barth and K. Kern, Nano Lett., 2005, 5, 901-904.

24 H. Spillmann, A. Dmitriev, N. Lin, P. Messina, J. V. Barth and K. Kern, J. Am. Chem. Soc., 2003, 125, 10725-10728.

25 M. Böhringer, K. Morgenstern, W.-D. Schneider and R. Berndt, Angew. Chem., Int. Ed., 1999, 38, 821-823.

26 M. Böhringer, W.-D. Schneider and R. Berndt, Angew. Chem., Int. Ed., 2000, 39, 792-795.

27 V. Humblot, M. O. Lorenzo, C. J. Baddeley, S. Haq and R. Raval, J. Am. Chem. Soc., 2004, 126, 6460-6469.

28 M. C. Blüm, E. Ćavar, M. Pivetta, F. Patthey and W. D. Schneider, Angew. Chem., Int. Ed., 2005, 44, 5334-5337.

29 D. Écija, K. Seufert, D. Heim, W. Auwärter, C. Aurisicchio, C. Fabbro, D. Bonifazi and J. V. Barth, ACS Nano, 2010, 4, 4936-4942.

30 D. Bléger, D. Kreher, F. Mathevet, A. J. Attias, G. Schull, A. Huard, L. Douillard, C. Fiorini-Debuischert and F. Charra, Angew. Chem., Int. Ed., 2007, 119, 7548-7551.

31 Y. Yang and C. Wang, Chem. Soc. Rev., 2009, 38, 2576-2589.

32 W. Xu, R. E. A. Kelly, H. Gersen, E. Lægsgaard, I. Stensgaard, L. N. Kantorovich and F. Besenbacher, Small, 2009, 5, 1952-1956.

33 A. Dmitriev, H. Spillmann, M. Lingenfelder, N. Lin, J. V. Barth and K. Kern, Langmuir, 2004, 20, 4799-4801.

34 S. Katano, Y. Kim, H. Matsubara, T. Kitagawa and M. Kawai, J. Am. Chem. Soc., 2007, 129, 2511-2515.

35 S. M. Barlow, S. Louafi, D. Le Roux, J. Williams, C. Muryn, S. Haq and R. Raval, Langmuir, 2004, 20, 7171-7176.

36 K. A. Simonov, N. A. Vinogradov, A. S. Vinogradov, A. V. Generalov, E. M. Zagrebina, G. I. Svirskiy, A. A. Cafolla, T. Carpy, J. P. Cunniffe, T. Taketsugu, A. Lyalin, N. Mårtensson and A. B. Preobrajenski, ACS Nano, 2015, 9, 8997-9011.
37 P. Han, K. Akagi, F. F. Canova, H. Mutoh, S. Shiraki, K. Iwaya, P. S. Weiss, N. Asao and T. Hitosugi, ACS Nano, 2014, 8, 9181-9187.

38 F. Schulz, P. H. Jacobse, F. F. Canova, J. van der Lit, D. Z. Gao, A. van den Hoogenband, P. Han, R. J. M. Klein Gebbink, M.-E. Moret, P. M. Joensuu, I. Swart and P. Liljeroth, J. Phys. Chem. C, 2017, 121, 2896-2904.

39 F. Esch, C. Dri, A. Spessot, C. Africh, G. Cautero, D. Giuressi, R. Sergo, R. Tommasini and G. Comelli, Rev. Sci. Instrum., 2011, 82, 53702.

40 For information on commercial availability of the FAST module contact ilo@elettra.eu.

41 F. J. Giessibl, Appl. Phys. Lett., 2000, 76, 1470-1472.

42 T. R. Albrecht, P. Grütter, D. Horne and D. Rugar, J. Appl. Phys., 1991, 69, 668-673.

43 L. Gross, F. Mohn, N. Moll, P. Liljeroth and G. Meyer, Science, 2009, 325, 1110-1114.

44 J. Cai, P. Ruffieux, R. Jaafar, M. Bieri, T. Braun, S. Blankenburg, M. Muoth, A. P. Seitsonen, M. Saleh, X. Feng, K. Müllen and R. Fasel, Nature, 2010, 466, 470-473.

45 A. Batra, D. Cvetko, G. Kladnik, O. Adak, C. Cardoso, A. Ferretti, D. Prezzi, E. Molinari, A. Morgante and L. Venkataraman, Chem. Sci., 2014, 5, 4419-4423.

46 Q. Fan, C. Wang, Y. Han, J. Zhu, J. Kuttner, G. Hilt and J. M. Gottfried, ACS Nano, 2014, 8, 709-718.

47 W. Wang, X. Shi, S. Wang, M. A. Van Hove and N. Lin, J. Am. Chem. Soc., 2011, 133, 13264-13267.

48 M. Koch, M. Gille, A. Viertel, S. Hecht and L. Grill, Surf. Sci., 2014, 627, 70-74.

49 M. Di Giovannantonio, M. El Garah, J. Lipton-Duffin, V. Meunier, L. Cardenas, Y. Fagot Revurat, A. Cossaro, A. Verdini, D. F. Perepichka, F. Rosei and G. Contini, ACS Nano, 2013, 7, 8190-8198.

50 J. Wintterlin, J. Trost, S. Renisch, R. Schuster, T. Zambelli and G. Ertl, Surf. Sci., 1997, 394, 159-169.

51 S. Berner, M. Brunner, L. Ramoino, H. Suzuki, H. J. Güntherodt and T. A. Jung, Chem. Phys. Lett., 2001, 348, 175-181.

52 D. G. de Oteyza, P. Gorman, Y.-C. C. Chen, S. Wickenburg, A. Riss, D. J. Mowbray, G. Etkin, Z. Pedramrazi, H.-Z. Z. Tsai, A. Rubio, M. F. Crommie and F. R. Fischer, Science, 2013, 340, 1434-1437.

53 F. Albrecht, N. Pavliček, C. Herranz-Lancho, M. Ruben and J. Repp, J. Am. Chem. Soc., 2015, 137, 7424-7428.

54 U. Schlickum, R. Decker, F. Klappenberger, G. Zoppellaro, S. Klyatskaya, M. Ruben, I. Silanes, A. Arnau, K. Kern, H. Brune and J. V. Barth, Nano Lett., 2007, 7, 3813-3817.

55 N. Henningsen, R. Rurali, C. Limbach, R. Drost, J. I. Pascual and K. J. Franke, J. Phys. Chem. Lett., 2010, 2, 55-61.

56 Z. Feng, S. Velari, A. Cossaro, C. Castellarin-Cudia, A. Verdini, E. Vesselli, C. Dri, M. Peressi, A. De Vita and G. Comelli, ACS Nano, 2015, 9, 8697-8709.

57 C. Bronner, J. Björk and P. Tegeder, J. Phys. Chem. C, 2015, 119, 486-493.

58 O. Voznyy, J. J. Dubowski, J. T. Yates and P. Maksymovych, J. Am. Chem. Soc., 2009, 131, 12989-12993. 
59 C. Africh, L. Köhler, F. Esch, M. Corso, C. Dri, T. Bucko, G. Kresse and G. Comelli, J. Am. Chem. Soc., 2009, 131, 3253-3259.

60 L. L. Patera, F. Bianchini, G. Troiano, C. Dri, C. Cepek, M. Peressi, C. Africh and G. Comelli, Nano Lett., 2015, 15, 56-62.

61 K. H. Ernst, Acc. Chem. Res., 2016, 49, 1182-1190.
62 C. Busse, S. Weigelt, L. Petersen, E. Lægsgaard, F. Besenbacher, T. R. Linderoth, A. H. Thomsen, M. Nielsen and K. V. Gothelf, J. Phys. Chem. B, 2007, 111, 5850-5860.

63 S. Kawai, A. S. Foster, T. Björkman, S. Nowakowska, J. Björk, F. Federici Canova, L. H. Gade, T. A. Jung and E. Meyer, Nat. Commun., 2016, 7, 11559. 